\title{
Activity Theory Based Ontology Model for Efficient Knowledge Sharing in eHealth
}

\author{
Yang Guo, Guohua Bai, Sara Eriksén \\ Blekinge Institute of Technology, Karlskrona, Sweden \\ Email: yang.guo@bth.se, guohua.bai@bth.se, sara.eriksen@bth.se
}

How to cite this paper: Guo, Y., Bai, G.H. and Eriksén, S. (2017) Activity Theory Based Ontology Model for Efficient Knowledge Sharing in eHealth. E-Health Telecommunication Systems and Networks, 6, 31-45.

https://doi.org/10.4236/etsn.2017.62003

Received: April 3, 2017

Accepted: June 17, 2017

Published: June 21, 2017

Copyright $\odot 2017$ by authors and Scientific Research Publishing Inc. This work is licensed under the Creative Commons Attribution International License (CC BY 4.0).

http://creativecommons.org/licenses/by/4.0/

\begin{abstract}
Knowledge sharing has become an important issue that challenges the efficient healthcare delivery in eHealth system. It also rises as one of the most demanding applications with reference to dynamic interactivities among various healthcare actors (e.g. doctors, nurses, patients, relatives of patients). In this paper, we suggest an activity theory based ontology model to represent various healthcare actors. The goal of the suggested model is to enhance interactivities among these healthcare actors for conducting more efficient knowledge sharing, which helps to design eHealth system. To validate the feasibility of suggested ontology model, three typical use cases are further studied. A questionnaire based survey is carried out and the corresponding survey results are reported, together with the detailed discussions.
\end{abstract}

\section{Keywords}

eHealth, Knowledge Sharing, Activity Theory, Ontology Model

\section{Introduction}

Over the recent decades, eHealth has played an important role in carrying out healthcare delivery and development on healthcare system. eHealth is referred to as the system with different applications of information and communications technology (ICT) that can support healthcare services [1]. The benefits from eHealth for society are many, such as improving the quality of healthcare services, expanding access to diagnostic services, increasing the efficiency of service delivery, reducing the cost of healthcare, contributing to the economies [2]. The underlying idea of eHealth is to practically conduct the healthcare services by applying electronic processes and communication into different healthcare actors. Here, healthcare actors are the individual persons or organizations that have impacts on or are affected by eHealth systems. Both healthcare providers and healthcare receivers are healthcare actors. 
Knowledge sharing has become a very important resource when knowledge needed for conducted services is extended outside of its own organization [3]. By eHealth, the diagnosis and treatment knowledge need to be shared between patients and those who may be concerned, like the healthcare providers. Meanwhile, with the healthcare network set up, healthcare receivers can share their experience and treatment solutions online with those who may be suffering from similar symptoms. With such practice applied, the efficiency in healthcare can be highly improved. In eHealth, sharing knowledge can realize potential gains and is critical to surviving and prospering in a competitive environment [4]. Knowledge sharing in eHealth has become crucial since health practitioners are required to be research-oriented, creative in healthcare, and ready to take new medical knowledge opportunities that can be acquired through various organizational learning mechanisms [5].

A very important problem in eHealth is that the various services and applications in different organizations use different vocabulary, concepts, models and so on, which leads to the problem of interoperability and the difficulty of sharing knowledge. To deal with this problem, it is very crucial for eHealth system designers to find a strategy to deal with the diversities in healthcare knowledge system development. A shared ontology may be a necessity to start with this strategy. Ontology, from technological aspect, is "an explicit machine-readable specification of a shared conceptualization" [6]. It is also a semantics approach to address the kinds and structures of objects, properties, events, processes and relations among these entities [7]. The goal of applying the ontology in eHealth is to establish a common semantics for addressing the concept of healthcare services. Corresponding entities in the healthcare ontology consist of not only the healthcare actors but also the knowledge and efforts involved in designing the eHealth system. Ontology may be utilized to promote knowledge sharing within organizations or inter-organizations.

During the past decade, the healthcare ontology has been widely investigated and developed. For instance, one standardization effort of Health Level Seven (HL7) is laid on developing the Service-Oriented Architecture (SOA) based healthcare ontology. The goal of this effort is to bridge standard ontologies in the domains like enterprise architecture, clinical care, and biomedicine [8]. Based on the work done by HL7, the authors of [3] report on the analysis of SOA based healthcare ontology by using a well-founded ontological approach called Unified Foundational Ontology for Service (UFO-S) [9]. The purpose of this work is to provide an ontological foundation to the SOA based healthcare ontology suggested by HL7. In [10], the authors study on the problem of representing the healthcare ontology from different perspectives associated with various healthcare actors and with using different terminologies. This work is motivated by a fact that it is difficult to creating a single universal ontology for the eHealth system.

So far, there are a large amount of studies done on knowledge sharing in the eHealth area. For instance, the authors of [11] report a multi-viewpoint based 
model to represent the heterogeneous health actors by using ontology. In [12], the authors report on the problem of distributed knowledge sharing among patients and physicians. They also suggest a multi-agent based method to overcome this problem. Clearly, the focus of these studies is laid on the representation of different health actors. The corresponding solution approach for knowledge sharing is mainly operated on two traditional heath actors. They are the care provides (e.g., doctors, nurses) and the care receivers (e.g., patients). Given the increasing demand for self-care on personal health problems, the care receivers may require the external assistant from other health actors like, e.g., family members, neighbors, social care agencies, third party organizations. This situation gives rise to the need of the long term financial sustainability on coordinating various healthcare actors. The motivation comes down to the development of solution approach to enhance the inter-activities among different health actors. The goal is to support more general knowledge sharing among the aforementioned various health actors, and thus improving the quality and efficiency of the assistant in self-care. To the best of our knowledge, there are few studies done so far along with this research line.

\section{Methodology}

To meet the requirement on the above-mentioned goal, in this paper we put the interest in investigating the interactivities among different healthcare actors in eHealth system.

- Firstly, the fundamental concepts related to healthcare ontology and activity theory are presented, together with the report on the related work.

- Secondly, based on activity theory we have proposed a new ontology model to represent dynamic interactivities of different healthcare actors, which are involved in the considered use cases to conduct knowledge sharing.

- Thirdly, to validate the feasibility of applying the suggested model in the considered use cases, a questionnaire based survey is carried out. Four different sets of questions are therefore distributed to four particular healthcare actors, i.e., patients, doctors, nurses, and relatives of patients.

- Finally, the corresponding survey results are reported. The detailed discussions are also presented to further analyze how the suggested model can practically enhance the interactivity of knowledge sharing in the considered use cases.

\section{Activity Theory Based Ontology Model}

\subsection{Activity Theory in eHealth}

The cultural-historical theory of activity, also known as Activity Theory (AT), is a framework of knowledge that seeks to explain the unity and inseparability of subjective mind and the objective human practice [13]. The origins of the theory can be found in the work of the Russian developmental psychologist Lev Vygotsky [14], Aleksei Nikolaevich Leontev [15], and especially further expanded by Engeström [16]. In Activity Theory, activity is the basic unit that preserves the essential quality behind any human practices. Engeström developed a trian- 
gle model based on previous work by Vygotsky and Leontev to recapitulate and visualize the components and relationships that compose an activity (Figure 1). A philosophical discussion about the model and relationship can be found in the study of [16], and will not be discussed here. We just take up the points which will contribute to the purpose of constructing ontology for eHealth system.

An activity is always conducted by some goal-oriented actors, a subject, directed towards an object or outcome that is being transformed. According to the theory a basic feature of human activity is the use of artefacts/instrument as mediation. Much of human interaction with the world is mediated through various artefacts/instrument. This triple relationship is represented in the top part of the triangle (Subject-Tool-Object-Outcome). The subject and object are related to and members of a community that is shown in the bottom part of the triangle. The Division of Labour between the Object and the Community is to specify the responsibility of the Object's all needs. The Rules are to legitimize the actions of the subject actors involved in the activity [17].

We specialize the work activity of a doctor (Subject) working at a healthcare centre for patients (Object). The outcome of the activity is to cure the patients from their health problems and illnesses. The instruments include such tools as EHR, X-rays, laboratory, and medical devices-as well as partially internalized diagnostic knowledge and treatment-related concepts and methods. A community is commonly considered a social unit (a group of people) who have something in common, such as norms, values, identity, and often a sense of place that is situated in a given geographical area (e.g. a village, town, or neighborhood). In healthcare area, the community consists of the staff of the clinic, other collaborating clinics and hospitals, municipality's home nurses. The division of labor determines the tasks and decision-making of the physician, the nurse, the nurse's aide, and other employee categories. Finally, the rules regulate the legislate actions taken by the doctor, use of time, the measurement of outcomes, the criteria for rewards and so on.

If we take the point of view of another subject in the community, for instance a nurse in the same healthcare activity, the model will keep the same, however, only the contents will be different. Also, if we take the patient as the subject of the activity system, their object will be the healthcare providers, which include doctors, nurses and other staff in healthcare center. Still, they share the same ar-

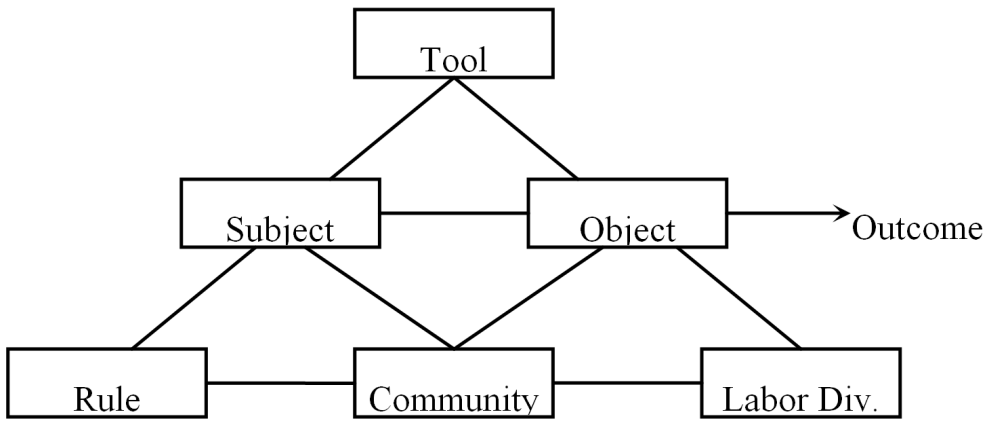

Figure 1. Engström's model of activity system. 
chitecture/model. This property of the model that is applicable in different levels and for multiple actors is a recursive property. Recursive property of a model can be used for constructing a system for multiple users in different organization levels which is exactly the case for eHealth system.

\subsection{System Description}

Based on the above-addressed activity theory and healthcare ontology, we consider a particular eHealth system, which is to provide personal healthcare services to elderly people. In this system, six different healthcare entities (i.e., healthcare actors and healthcare elements) are taken into consideration. They are described below:

- Information about care receivers: it is related to the personal information about the care receivers for the self-care assistant purposes. Examples of such information are the age, the suffered chronic diseases, and the relatives (i.e., family members). All these information will be recorded in this database of care receivers. Further, the information about the associated care providers is also required to be recorded in this database.

- Information about care providers: similarly, it is related to the personal information about the care providers. Examples of such information are the time scheduling of care providers, the competence of care providers, and the information about the assigned care receivers. All these information will be recorded in this database of care providers.

- Instruments: they are referred to as the artefacts used for personal healthcare services, for instance, a laboratory, the electronic health record, the X-rays and the relevant diagnostic knowledge. These items need to be integrated into a single base, which provides the uniform Application Programming Interfaces (APIs) to different categories of users such as healthcare providers and receivers.

- Community network: it is the social context which includes all actors involved in the activity system. The community network is connected to different social actors and elements like, e.g., patients' relatives, family members, healthcare centers, shopping centers, banks, TAXI etc.

- Laws/rules: they are conventions, guidelines and rules regulating activities in the system. They are used by different actors to know the relevant information about the particular social laws, contracts, and decisions.

- Labour division: it is the social hierarchical structure of activity, the division of activities among actors in the system. Labour division provides the prescribed social contact information for dealing with personal healthcare, together with the suitable methods to solve the particular conflict problem among different healthcare actors.

\subsection{Ontology Model Description}

In accordance with the triangle model advanced in [16], we suggest a new model to represent the above-described system. This model is based on both activity theory and healthcare ontology, and thus so-called Activity Theory based On- 
tology (ATO) model. The main advantage of this model is referred to as the flexibility and extendibility of investigating the interactivities among different healthcare actors. The goal of using this model is to enhance the efficiency of conducting knowledge sharing in more general eHealth system. The detailed description about the suggested model is as follows.

In Figure 2, among six activity units, there are three key ones, i.e., care provider, care receiver and tool box. First, the care provider and the care receiver are defined to be the activity subject and activity object, respectively. The work activity of the care provider is then referred to as the assistant in the self-care requested by a particular care receiver. The outcome of this work activity is connected to the process of dealing with the particular health problem suffered by the particular care receiver. To accomplish such process, one needs the particular instruments or so-called tool box such as laboratory, medical devices (e.g., electronic health record, X-rays), relevant diagnostic knowledge, and the suitable psychological treatment methods. Further, all these instruments are provided by the tool box.

The above-described three key activity units are further extended by connecting them to another three activity units. The first unit is related to the community network, which is connected to both the care providers and receivers. This is because that different social contacts belonging to this community may need collaborate with care providers to give more efficient healthcare service to care receivers. The second unit is the division of labor, which is responsible for doing decision-making on the particular tasks in the eHealth system. Examples of tasks are assigning the competent physician for the patient, allocating the nurse to the patient, and arranging meetings between care providers and care receivers. Due to the limited resource on the care provider side, the division of labor is designed to connect to the community unit. The third unit is the associated with the prescribed rules, which give the particular constraints to different actors who involved in the activity like, e.g. the legislate actions taken by care providers, the outcome evaluations, the reward criteria for physicians.

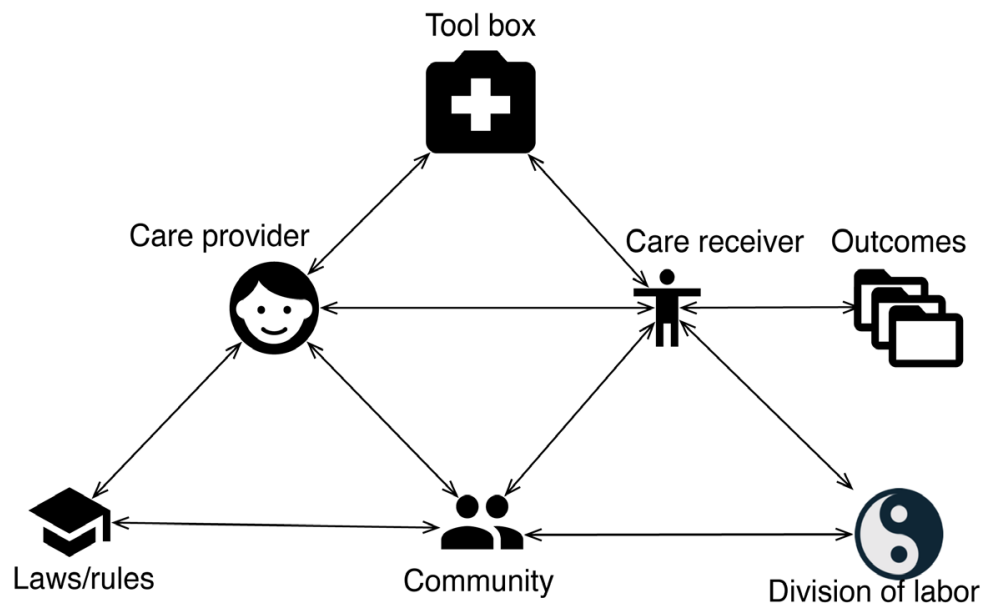

Figure 2. Activity theory ontology model for knowledge sharing in eHealth. 


\section{Model Validation}

\subsection{Use Cases}

To validate the feasibility and effectiveness of the suggested model, one needs to apply it to the practical healthcare system. To do this, we have conducted the preliminary observation on the typical use cases existing in the real healthcare system. The observation was done at the Zhengzhou University Hospital, which is located in the city of Zhengzhou in China. The reason for selecting this hospital is that, the population of elderly people in the city of Zhengzhou is larger than the other major cities in China. Such situation leads to various interactivities among various healthcare actors during healthcare service delivery process.

The observation results show that there are three typical use cases: i) a patient at home communicates with a nurse; ii) a family member takes care of a patient; iii) a patient makes an appointment to meet a doctor. We then apply the suggested ATO model to these three use cases. Accordingly, the newly formalized representation of interactivities among different healthcare entities (i.e., actors and elements) are illustrated Figures 3-5, together with the brief descriptions.

Use case 1: a patient communicates with a nurse

Number (1), (2), etc. are according to the numbers in Figure 3.

(1): A particular patient stays at home and feels bad. As such, this patient reports the health status to a nurse, who is responsible for assisting this patient.

(2): The nurse checks the information about the health status reported by the patient. Depending on the particular condition of the health status, the nurse may give different feedbacks to the patient.

(3): If the situation is not serious, the nurse may directly give the corresponding suggestion to the patient. If the situation is serious, the nurse immediately reports the patient's health status to a doctor who is responsible for taking care of the patient.

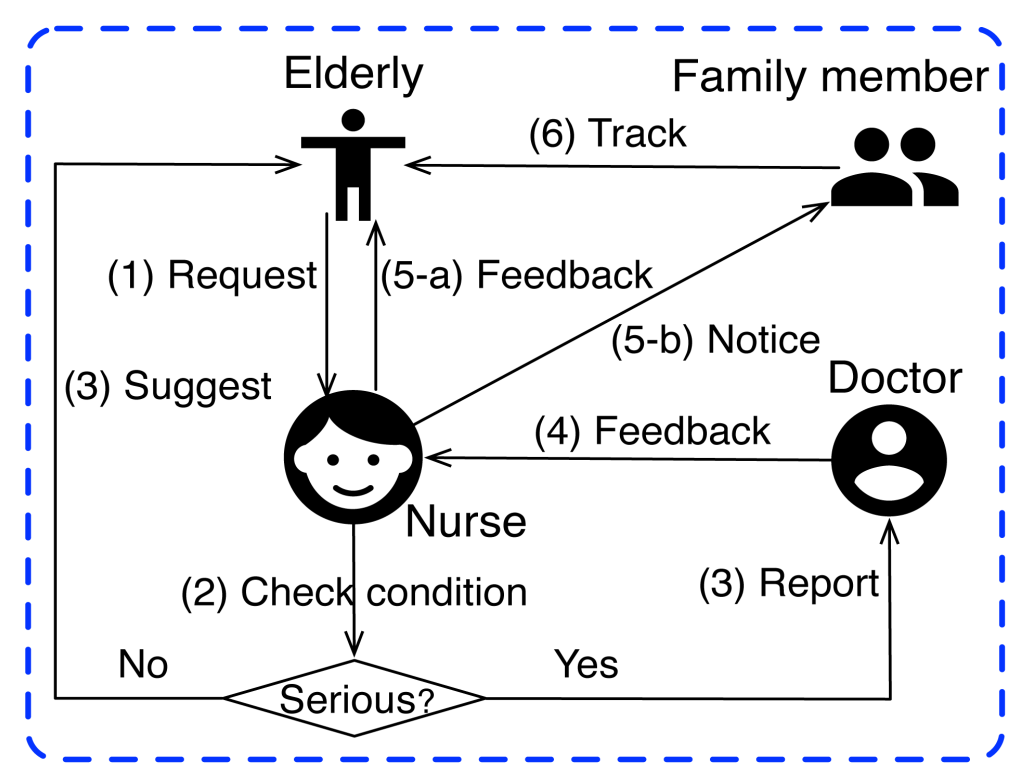

Figure 3. Activity theory ontology model for case 1 . 


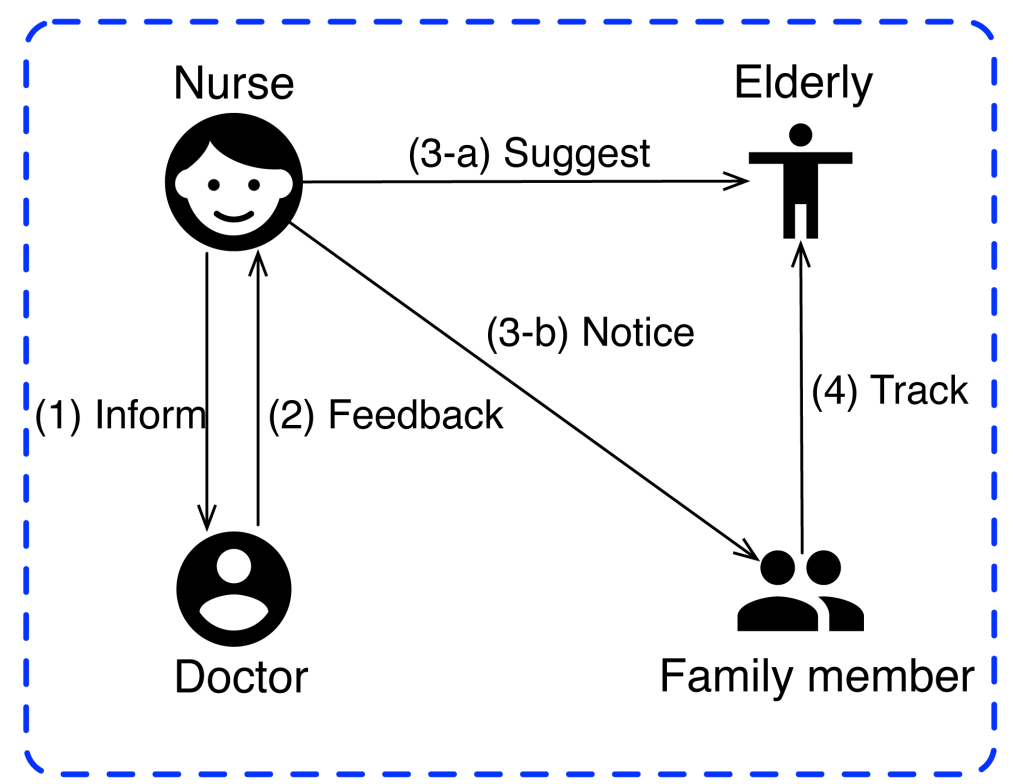

Figure 4. Activity theory ontology model for case 2.

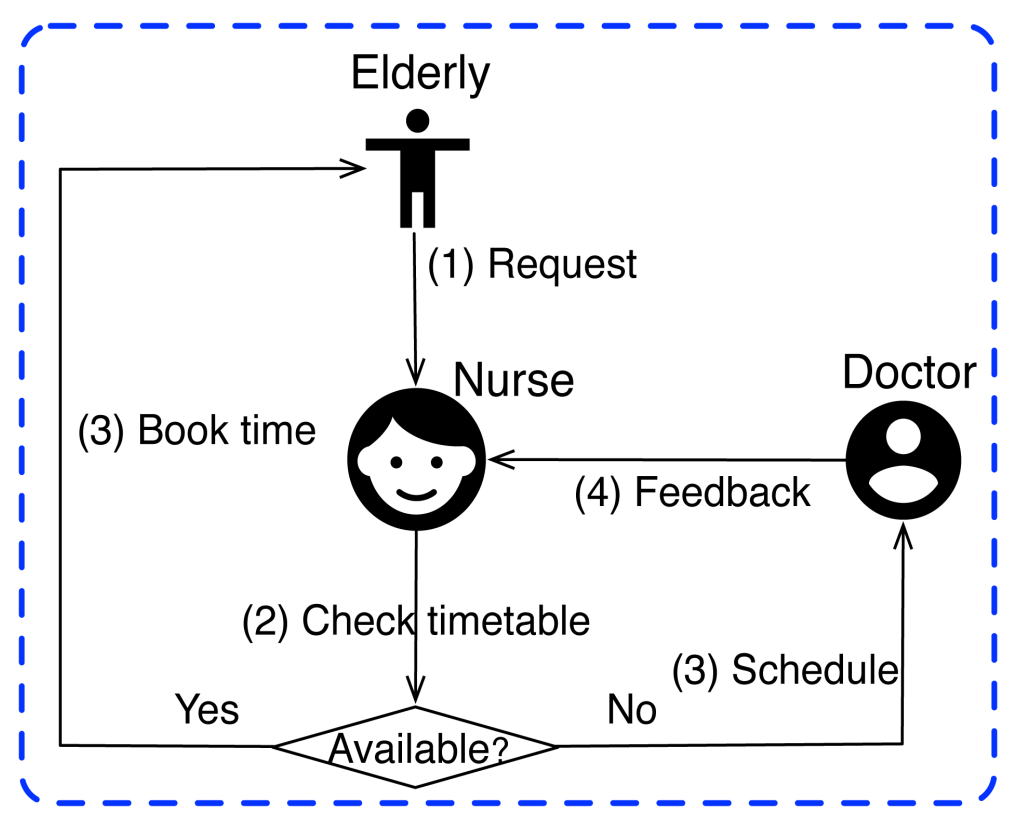

Figure 5. Activity theory ontology model for case 3.

(4): The doctor makes a further investigation on the reported health status. The doctor also makes a prescription for the patient, and sends the prescription to the nurse.

(5): The nurse provides the received prescription to the patient. Meanwhile, the nurse also sends the notice to the patient's family member.

(6): The patient's family member could help the nurse to keep tracking the health status of the patient.

Use case 2: a family member takes care of a patient

Number (1), (2), etc. are according to the numbers in Figure 4.

(1): A nurse checks the health status of a patient, which is reported by a pa- 
tient's family member. The nurse observes that the patient's health status is not good, and thus informing a doctor who is responsible for taking care of the patient.

$(2,3,4)$ : They are same to the steps $(4,5,6)$ described in case 1 .

Use case 3: a patient makes an appointment to meet a doctor

Number (1), (2), etc. are according to the numbers in Figure 5.

(1): A particular patient sends an appointment request to a particular nurse who is responsible for assisting this particular patient.

(2): The nurse checks the time availabilities of the corresponding doctor, and sends the suggested time slots to this doctor.

(3): Based on the received information about the time slots suggested by the nurse and the appointment requested by the patient, the doctor arranges a particular time slot to meet the patient. The information about this particular time slot is then sent to the nurse.

(4): Once the nurse receives the arranged time slot for meeting from the doctor, the nurse informs this time slot to the patient and prepares for the meeting between the doctor and the patient.

\subsection{Questionnaire Based Survey}

The above described three use cases show the potential possibility of applying the ATO model in eHealth system. However, the implementation feasibility of this model and the assumed effectiveness of dealing with healthcare service still need to be preliminary validated in accordance with the practical demands from different healthcare actors. To do kthis, the questionnaire based survey is conducted.

The survey was carried out between 2016 March and April at the Zhengzhou University Hospital (the same hospital we mentioned before). The survey goals mainly include four categories of healthcare actors represented in the ATO model. They are the healthcare providers (e.g., the doctors and nurse working in the hospital), the healthcare receivers (e.g., the elderly people staying at home), the community (e.g., the patients' relatives or family members) and the tool box (e.g., laboratory in the hospital). Examples of the considered interactivities among them are booking a meeting appointment with a nurse and/or a doctor, conducting the examination on the healthcare status of patients, reporting outcomes by either nurses or by doctors, checking results by either patients or by their relatives.

Further, four sets of questions are distributed to four groups of participants, respectively. They are accordingly referred to as Group of patients, Group of doctors, Group of nurses, and Group of patients' relatives. For the simplicity purpose, the four groups of participants are labelled as G-p, G-d, G-n and G-r in the following presentation.

The focus of the questionnaire based survey is to enhance interactivities among these health actors for conducting more efficient knowledge sharing, which is helping design eHealth system. The suggested ATO model and the con- 
sidered use cases indicate that designing efficient knowledge sharing in eHealth system is a sophisticated procedure due to heterogeneous healthcare actors and their interactivities involved in healthcare service delivery. To alleviate the complexity of this procedure, a feasible solution approach is to redefine the responsibilities of those healthcare actors with reference to their particular roles of assisting the particular interactivities. The main goal of this approach is to help healthcare providers (e.g., doctors and nurses) to have the better understanding of the real need from patients from both medical treatment and psychology perspectives. As such, they can improve the efficiency of carrying out the corresponding interactivities with patients and other healthcare actors (e.g., the relatives of patients), and thus delivering better healthcare services to patients.

Subsequently, the above-mentioned four sets of questions need to be well formalized with the aim at identifying and developing the necessary responsibilities for various healthcare actors. Along with this line, 12 key questions are reported in Figures 6-9, together with corresponding survey results.

\subsection{Result and Analysis}

For group G-p, there are 40 patients participating in the survey with the given questionnaires. The questionnaires for groups G- $d$ and G-n are distributed to 25 doctors and 35 nurses, respectively. Similarly, the questionnaires for group G-r are distributed to 40 people. They are the relatives of the patients in group G-p. Eventually, 35 results are received from the group G-p. While, 23 and 32 results are received from groups G-d and G-n, respectively. Further, 33 results are received from group G-r. In other words, the response rates to the survey are about $87.5 \%, 92.0 \%, 91.4 \%$ and $82.5 \%$ for the patients, doctors, nurses and relatives, respectively. The survey results for all the above-listed questions are reported in Figures 6-9. The discussions about these results are described below.

In Figure 6, the answers to question Q-p-1 show that the individual patient usually wants to discuss with the doctor about their potential illness when he/she
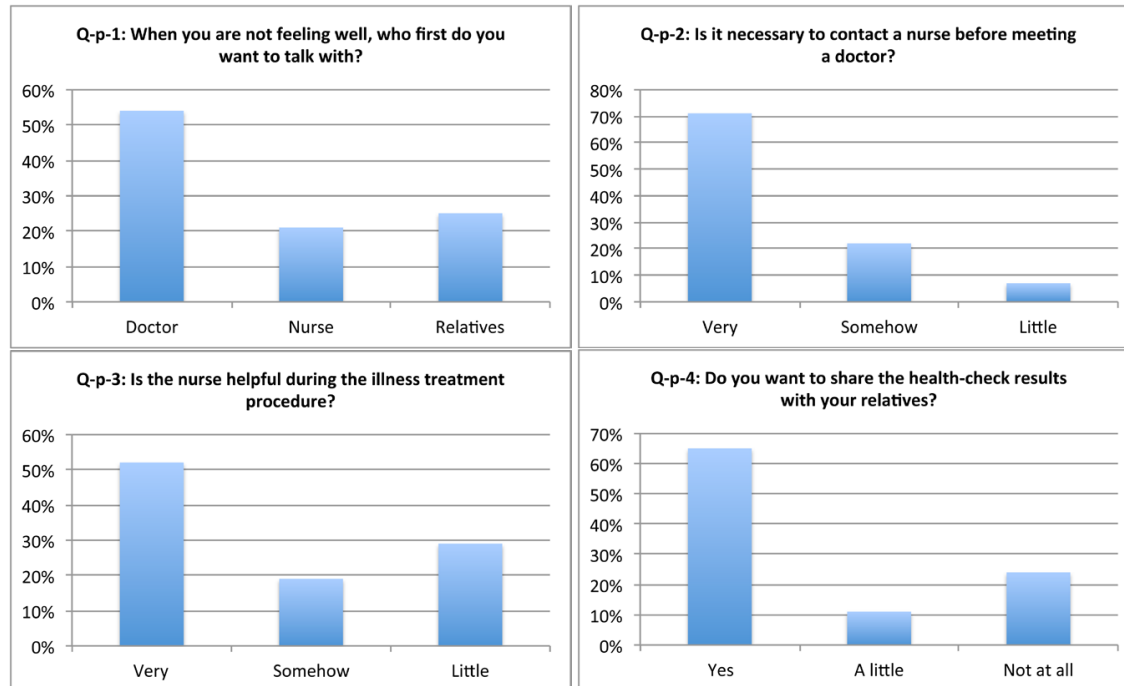

Q-p-4: Do you want to share the health-check results with your relatives?

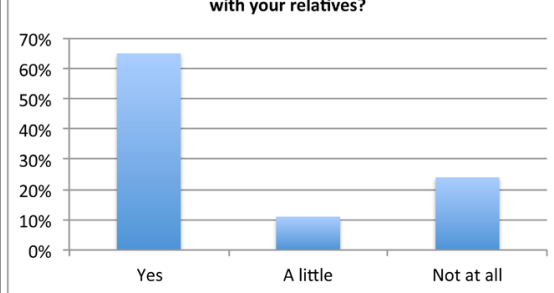

Figure 6. Survey results for group G-p questions. 

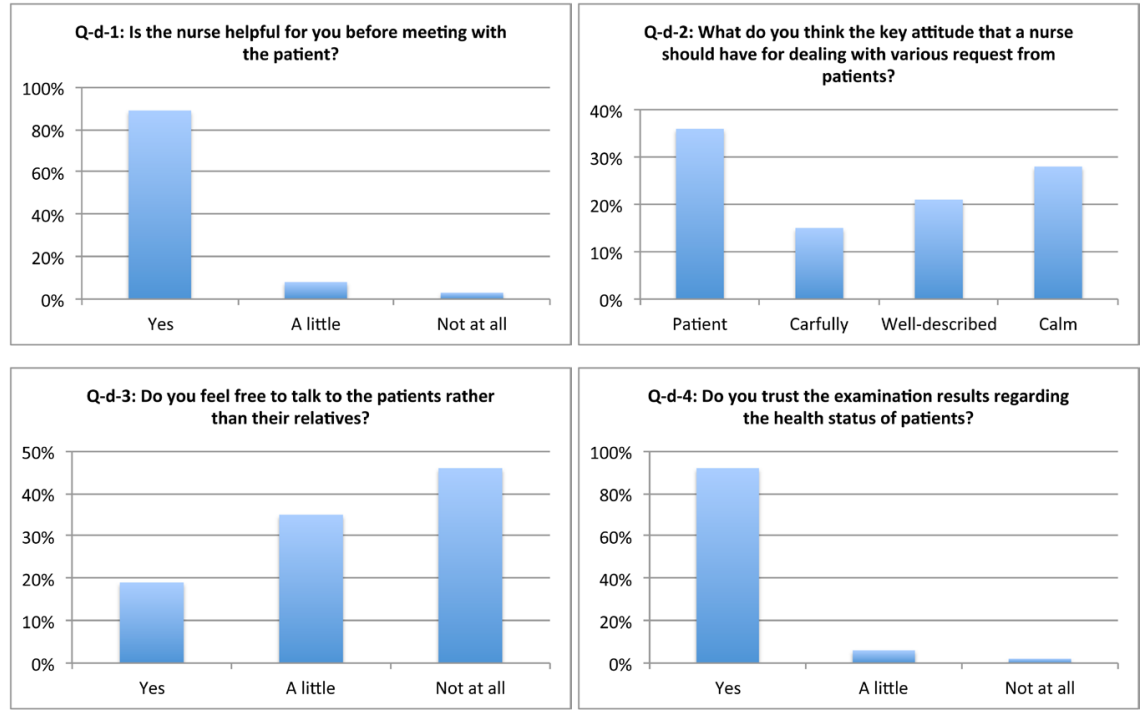

Figure 7. Survey results for group G-d questions.
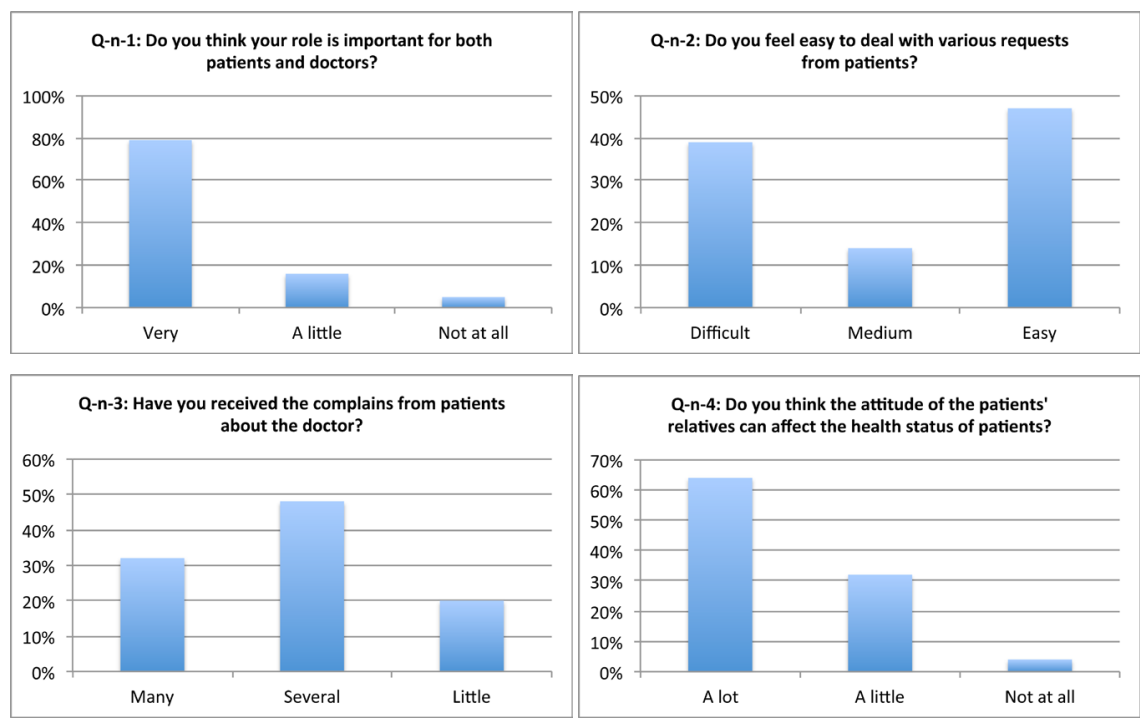

Figure 8. Survey results for group G-n questions

does not feel well. However, the doctor may be not available for the direct contact demanded by the patient due to the constrained time scheduling. In this case, the nurse becomes an important role who can take the responsibility of dealing with various requests from patients. Based on the preliminary evaluation on the health status of patients, if the meeting between the doctor and the nurse is needed, the nurse can further arrange such a meeting according to the time availabilities of both doctor and patient. Such preliminary evaluation is highly necessary by both the doctor and the patient, as shown by the answers to questions Q-p-2 and Q-d-1 in Figure 6 and Figure 7. As shown by the answers to question Q-d-3 in Figure 7, the patient also thinks that the nurse is also helpful during the illness treatment procedure.

As shown by the answers to Q-n-1 in Figure 8, most of nurses agree with that their responsibility of bridge communication gap between doctors and patient is 
very important to the healthcare delivery service. The biggest problem faced by the nurse is connected to the need of coping with various requests demanded by patients, including the expected complains about the doctor, as shown by the answers to questions Q-n-2 and Q-n-3 in Figure 8. To alleviate this problem, the doctors suggest that the nurses should have several important attitudes to patients, such as being patient and maintaining calm, as shown by answers to question Q-d-2 in Figure 7.

In Figure 9, the answers to questions Q-d-1 and Q-d-2 show that, although the patient's relatives are care about the health status of the patients, they may lack time to provide the professional healthcare to the patient. This also indicates that the patients prefer to talk with the healthcare professions (i.e., the doctor) about their health status, as shown by answers to question Q-p-1 in Figure 6. However, the answers to question Q-p-4 show that the patients want to share the information about their health status to their relatives, as they definitely want to get the help from the relatives. Further, the relatives of patients also agree with that the nurse is easier to communicate rather than doctor, as shown by the answers to question Q-r-3.

\subsection{Discussions}

In this paper, to improve knowledge sharing efficiency in eHealth, we proposed ATO model to enhance the interactivities among different healthcare actors. The above considered use cases and corresponding survey results show the feasibility and effectiveness of the suggested model. Specifically, for a particular interactivity in eHealth, the proposed model can be used to redefine the particular responsibilities of healthcare actors, who are involved in conducting the particular interactivity. Such redefined responsibilities can then contain the information about how to efficiently accomplish the corresponding interactivities among different healthcare actors. Meanwhile, the efficiency of knowledge sharing among
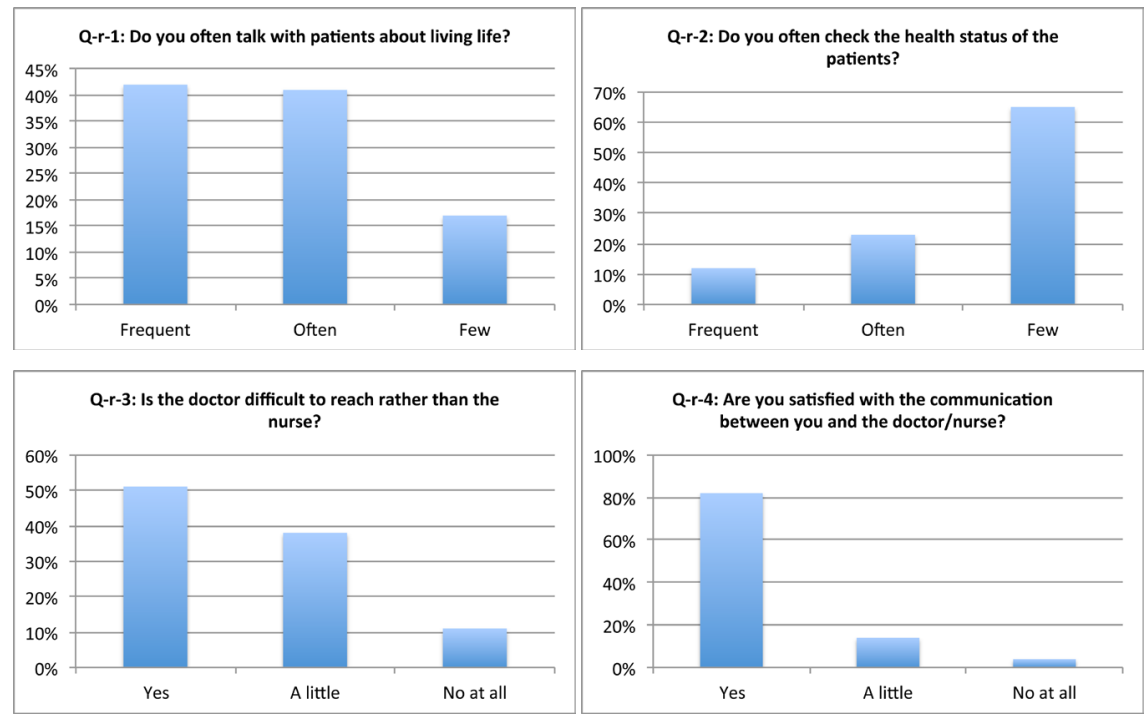

Figure 9. Survey results for group G-r questions. 
these healthcare actors is improved with reference to the efficient accomplishment of the related healthcare service delivery.

Regarding the aforementioned need of redefining the responsibilities of healthcare actors, the discussions are as follows.

The above-reported survey results show that knowledge sharing in eHealth system is a sophisticated procedure due to heterogeneous healthcare actors and their interactivities involved in healthcare service delivery. To alleviate the complexity of this procedure, one needs to redefine the responsibilities of those healthcare actors with reference to their assistant in accomplishing the associated interactivities.

For instance, an important interactivity in the healthcare system is regarding the examination on the illness of patients. The involved healthcare actors are like, e.g., the patient, the nurse, the doctor, the patient's relative and the laboratory (including e.g., medical devices). Due to the need of dealing with multiple patients, the doctor usually needs the information about the preliminary evaluation on the health status of individual patient. Based on evaluation results, the doctor then decides on whether or not carrying out examination by using the laboratory. In this case, the nurse becomes very important to take the role of doing such preliminary evaluation patients with reference to the historical information about the health status of patients. As show in Figure 6, such new role of the nurse is also required by patients as they prefer to first talk to the nurse about their health status rather than the doctor. This is because a particular doctor is likely difficult to reach by patients as the constraint on the doctor's time scheduling, as show in Figure 9.

Another important interactivity is connected to delivering the examination report from the doctor to the patient. In this interactivity, the involved healthcare actors should be referred to as not only the patient, the nurse and the doctor, but also the patient's relatives. From Figure 8, we observe that the patient's relatives can bridge the communication gap between the doctor and the patient. Specifically, the doctor is usually expert in the profession skills like, e.g., illness examination, medical treatment, summarizing examination results. However, the Figure 6 shows that when the examination results are delivered to the patient, more social communication is demanded by the patient. This is because the direct result report from the doctor to the patient may significantly affect the patient's emotion, and thus leading to the unexpected mental injury to the patient. In this case, the patient's relatives can accordingly provide the necessary psychological support to the patient.

The survey results also show other interesting interactivities, such as the time scheduling for both the doctor and the patient to do illness examination, coping with various requests and complains from patients, the social activities among patients. By using our suggested ATO model, all of these interactivities can be represented in an efficient way, based on which the responsibilities of the involved healthcare actors are redefined and the knowledge sharing among them is hence enhanced. 


\section{Conclusion}

In this paper, we studied the problem of coping with interactivity among different health actors for knowledge sharing in eHealth. The activity theory based ontology model is suggested for representing the interactivities among different health actors in the eHealth system. Based on three typical use cases, a questionnaire based survey is conducted. The goal is to validate the suggested model with reference to the practical requirements demanded by the real healthcare system. The results show the feasibility of the suggested model and the effectiveness of applying it to the considered use cases. The future work is to implement a prototype software system, based on which, the suggested model can be practically integrated and be used for improving the efficiency of knowledge sharing in eHealth.

\section{References}

[1] Jung, M.L. and Loria, K. (2010) Acceptance of Swedish e-Health Services. Journal of Multidisciplinary Healthcare, 3, 55-63.

[2] Stroetmann, K.A., Jones, T., Dobrev, A. and Stroetmann, V.N. (2006) eHealth Is Worth: The Economic Benefits of Implemented eHealth Solutions at Ten European Sites. Technical Report, European Commission.

[3] Milosevic, Z., Almeida, J.P.A.A. and Nardi, J.C. (2014) Towards Better Semantics for Services in eHealth Standards: A Reference Ontology Approach. IEEE 18th International Enterprise Distributed Object Computing Conference Workshops and Demonstrations, Ulm, Germany, 1-2 September 2014, 276-285.

https://doi.org/10.1109/edocw.2014.48

[4] O’Dell, C. and Grayson, C. (1998) If Only We Knew What We Know: Identification and Transfer of Internal Best Practices. California Management Review, 40, 154174. https://doi.org/10.2307/41165948

[5] Lipshitz, R. and Popper, M. (2000) Organizational Learning in a Hospital. The Journal of Applied Behavioral Science, 36, 345-361. https://doi.org/10.1177/0021886300363005

[6] Gómez-Pérez, A. and Corcho, O. (2002) Ontology Specification Languages for the Semantic Web. IEEE Intelligent Systems, 17, 54-60.

https://doi.org/10.1109/5254.988453

[7] Kalra, D. (2006) Archetypes: The Missing Link? EuroRec Annual Conference. http://www.eurorec.org/EuroRec2006/presentations/EuroRec2006 DipakKalra.ppt

[8] Health Level Seven. Healthcare SOA Ontology Project. http://www.hl7.org/special/committees/projman/searchableprojectindex.cfm?action $=$ edit $\&$ ProjectNumber $=628$

[9] Nardi, J.C., Falbo, R., Almeida, J.P.A., Guizzardi, G., Pires, L.F., van Sinderen, M. and Guarino, N. (2013) Towards a Commitment-Based Reference Ontology for Services. 17 th IEEE International EDOC Conference (EDOC), Vancouver, BC, 9-13 September 2013, 175-184. https://doi.org/10.1109/edoc.2013.28

[10] Kataria, P. and Juric, R. (2010) Sharing e-Health Information through Ontological Layering. 2010 43rd Hawaii International Conference on System Sciences (HICSS), Honolulu, HI, 5-8 January 2010, 1-10. https://doi.org/10.1109/hicss.2010.338

[11] Hemam, M. and Boufaida, Z. (2014) Towards a Multi-Viewpoints Ontological Model for Knowledge Sharing in E-health. International Journal of Information 
Science and Intelligent System, 3, 5-14.

[12] Widmer, T., Premm, M., Schuele, M., Murray, J. and Karaenke, P. (2013) Distributed Knowledge Sharing For Patient Guidance eHealth Services. 21 st European Conference on Information Systems, Utrecht, Netherlands.

[13] Engeström (1987) Learning by Expanding. An Activity-Theoretical Approach to Developmental Research. Orienta-Konsultit Oy, Helsinki, Finland.

[14] Vygotsky Lev, S. (1978) Mind in Society: The Development of Higher Psychological Processes. Harvard University Press, Cambridge, MA.

[15] Leontev, A.N. (1978) Activity, Consciousness and Personality. Prentice-Hall, Englewood Cliffs, NJ.

[16] Engeström (1990) Working, and Imagining: Twelve Studies in Activity Theory. Orienta-Consultit Oy, Helsinki.

[17] Zhang, P. and Bai, G. (2004) An Activity System Theory Approach to Agent Technology. 5 th International Symposium on Knowledge and Systems Sciences, Ishikawa, Japan.

Submit or recommend next manuscript to SCIRP and we will provide best service for you:

Accepting pre-submission inquiries through Email, Facebook, LinkedIn, Twitter, etc. A wide selection of journals (inclusive of 9 subjects, more than 200 journals)

Providing 24-hour high-quality service

User-friendly online submission system

Fair and swift peer-review system

Efficient typesetting and proofreading procedure

Display of the result of downloads and visits, as well as the number of cited articles

Maximum dissemination of your research work

Submit your manuscript at: http://papersubmission.scirp.org/

Or contact etsn@scirp.org 